\title{
Regulation of the Drosophila ubiquitin ligase DIAP1 is mediated via several distinct ubiquitin system pathways
}

\author{
Y Herman-Bachinsky ${ }^{1}$, H-D Ryoo ${ }^{2}$, A Ciechanover ${ }^{\star, 1}$ and H Gonen ${ }^{1}$
}

Inhibitors of apoptosis proteins (IAPs) suppress cell death by inactivating proapoptotic regulators, and therefore play important roles in controlling apoptosis in normal and malignant cells. Many IAPs are ubiquitin ligases, and their activity is mediated via ubiquitination and subsequent degradation of their targets. Here we corroborate a previous observation that DIAP1 (Drosophila IAP1) can be degraded via a two-step mechanism: (i) limited caspase-mediated cleavage and (ii) degradation of the released fragment via the ubiquitin N-end rule pathway. Yet, we demonstrate that this pathway is not the only one involved in DIAP1 degradation, and the intact protein can be degraded independent of prior caspase cleavage. Importantly, this mode of degradation does not require the RING-finger-mediated autoubiquitinating activity of DIAP1, believed to target many RING-finger E3s for self-destruction. Our preliminary data suggest that DIAP2 mediates DIAP1 degradation, suggesting a novel regulatory loop within the apoptotic pathway. Studying the role of the autoubiquitinating activity of DIAP1, we demonstrate that it does not involve formation of Lys48-based polyubiquitin chains, but probably chains linked via Lys63. Our preliminary data suggest that the autoubiquitination serves to attenuate the ligase activity of DIAP1 towards its exogenous substrates.

Cell Death and Differentiation (2007) 14, 861-871. doi:10.1038/sj.cdd.4402079; published online 5 January 2007

Recent studies indicate that ubiquitination plays an important role in regulating apoptosis. This is partially mediated by the inhibitors of apoptosis proteins (IAPs), a centrally important group of cell death regulators, many of them are ubiquitin ligases. IAPs suppress cell death by inactivating different proapoptotic factors, some by targeting them for ubiquitination and subsequent proteasomal degradation. At least eight IAPs have been identified in mammals. In Drosophila melanogaster, expression of DIAP1 and DIAP $2^{1}$ can suppress apoptosis that occurs both during normal development as well as following overexpression of proapoptotic factors such as Reaper (Rpr). ${ }^{2}$ Loss of DIAP1 function results in early embryonic death as a consequence of massive apoptosis. ${ }^{3-5}$ The IAP family is characterized structurally by one-three $\mathrm{N}$ terminal copies of Baculovirus IAP Repeat (BIR) domains. DIAP1 contains two copies of BIR that bind effector and initiator caspases. In some cases, this binding leads to ubiquitination and subsequent degradation of the caspase. ${ }^{2}$ IAP-mediated inactivation of caspases is effectively inhibited by a family of proapoptotic proteins that share an IAP-binding tetra-peptide motif at their $\mathrm{N}$-termini. Among those proteins are Smac/Diablo in mammals, and Rpr, Hid and Grim (RHG) in Drosophila. It was shown that RHG proteins abrogate effectively DIAP1-mediated inactivation of Dronc and DrICE. ${ }^{6}$ DIAP1 contains also a C-terminal RING-finger motif that serves to recruit the E2 component of the ubiquitin conjugation machinery. For RING-finger E3s, their best-characterized activity is self-ubiquitination thought to regulate their cellular level, but it is assumed that most of these ligases have also exogenous substrates. ${ }^{7,8}$ In Drosophila melanogaster, the E2 UbcD1 is the partner of DIAP1. ${ }^{9}$ Like some other RING-finger proteins, DIAP1 catalyzes its own ubiquitination, and this activity is stimulated dramatically by the RHG proteins. ${ }^{9-14}$ The currently known exogenous substrates of DIAP1 include the proapoptotic proteins Rpr and the caspases Dronc and DrICE. ${ }^{14,15}$ It has been shown that ubiquitination of $\mathrm{Rpr}$ leads to its degradation, which terminates its antiapoptotic function. ${ }^{15}$

Not much is known on DIAP2. It bears similarity to DIAP1 and has three $\mathrm{N}$-terminal BIR domains and a C-terminal RING-finger domain. Overexpression of DIAP2 suppresses Rpr- and Hid-, but not Grim-dependent cell death. ${ }^{16}$ However, unlike DIAP1, removal of DIAP2 does not enhance overexpressed Rpr phenotype, ${ }^{1}$ that because DIAP1 can efficiently regulate $\mathrm{Rpr}$. Of note is that unlike DIAP1, DIAP2 cannot interact with most Drosophila caspases. ${ }^{17,18}$

Ditzel et al. ${ }^{19}$ demonstrated that shortly after initiation of apoptosis in S2 cells, DIAP1 is cleaved after Asp20 by the effector caspase DrICE. They further showed that the resulting long C-terminal fragment is important for the antiapoptotic activity of DIAP1, and because it contains a newly exposed asparagine residue in the $\mathrm{N}$-terminal position, it is recognized and rapidly degraded via the $\mathrm{N}$-end rule pathway of the ubiquitin system. The $\mathrm{N}$-end rule relates the

\footnotetext{
${ }^{1}$ Cancer and Vascular Biology Research Center, The Rappaport Faculty of Medicine and Research Institute, Technion-Israel Institute of Technology, Haifa, Israel and ${ }^{2}$ Department of Cell Biology, New York University School of Medicine, New York, NY, USA

${ }^{*}$ Corresponding author: A Ciechanover, Cancer and Vascular Biology Research Center, The Rappaport Faculty of Medicine and Research Institute, Technion-Israel Institute of Technology, Efron Street, Bat Galim, PO Box 9649, Haifa 31096, Israel. Tel: + 9724829 5379/5427/5356; Fax: + 97248521193 ;

E-mail: c_tzachy@netvision.net.il

Keywords: IAP; ubiquitin; degradation; N-end rule; apoptosis; Caspase; Drosophila

Abbreviations: IAPs, inhibitors of apoptosis proteins; BIR, Baculovirus IAP Repeat, RHG, Rpr, Hid and Grim

Received 05.9.06; revised 30.10.06; accepted 06.11.06; Edited by G Melino; published online 05.1.07
} 
in vivo half-life of a limited group of protein substrates to two groups of exposed residues at the $\mathrm{N}$-terminal position, 'stabilizing' and 'destabilizing'. ${ }^{20}$ Asparagine is not a 'destabilizing' residue as such, but becomes so following activity of asparginase that converts it to aspartate, which then undergoes modification by arginine,${ }^{21-25}$ that is recognized by a specific ubiquitin ligase E3 $\alpha$. Ditzel concludes that DIAP1 can be degraded via two distinct ubiquitin-mediated mechanisms: (i) RING-finger-mediated autoubiquitinating pathway ${ }^{15}$ and (ii) caspase-mediated processing followed by rapid degradation of the released fragment via the $\mathrm{N}$-end rule pathway. Yokokura et al. ${ }^{26}$ have also shown that apoptosis induces caspase-mediated processing of DIAP1, in the same site. However, in contrast to Ditzel et al, ${ }^{19}$ they found that the cleavage of DIAP1 promotes its further degradation via autoubiquitination, that requires the RING-finger domain of the protein. ${ }^{26}$ In addition, Yan et al. ${ }^{27}$ proposed that Dronc cleaves DIAP1 after $\mathrm{Glu}^{205}$, resulting in the generation of two separate functional units: (i) a BIR1-containing fragment that inhibits DrICE by binding to it and (ii) a BIR2-RING fragment that can bind Dronc and ubiquitinate it. ${ }^{27}$ According to these findings, since the initiator caspase Dronc is probably activated before the effector caspase DrICE, the N-end rule pathway will be involved, if at all, in the removal of the $\mathrm{N}$-terminal BIR1 domain-containing fragment (and even that after further removal of its first $20 \mathrm{~N}$-terminal residues by DrICE), but not in removing the BIR2-RING fragment. Ryoo et al. ${ }^{9}$ showed that Rpr-induced removal of DIAP1 occurs in the presence of $\mathrm{p} 35$, a strong caspase inhibitor, suggesting that caspase cleavage is not essential for removal of DIAP1 under those conditions. Deveraux et al. ${ }^{28}$ and Clem et al. ${ }^{29}$ have shown an involvement of caspase cleavage in the regulation of mammalian IAPs. XIAP, a potent human IAP, is cleaved during Fas (CD95)-induced apoptosis into two fragments: (i) BIR1-BIR2 and (ii) BIR3-RING finger. In this case, it appears that the cleavage, which inactivates XIAP, serves as a mechanism by which the threshold of caspase activity necessary for inducing apoptosis is lowered. The human IAPs, clAP1 and clAP2, are less potent inhibitors of apoptosis compared to XIAP. They are cleaved during apoptosis and release their C-terminal RING domain. This cleavage appears to activate a latent antiapoptotic activity, probably via removal of the RING-finger domain that results in abrogation of the autoubiquitinating activity that would result in their subsequent degradation. Taken together, these data demonstrate that the activity of IAPs can be regulated by several distinct and apparently opposing mechanisms.

In this study, we demonstrate that DIAP1 degradation proceeds via at least two mechanisms, both mediated by the ubiquitin system: (i) the reported mechanism by which caspase-mediated processed DIAP1 is recognized and degraded via the $\mathrm{N}$-end rule pathway in a reaction that is mediated by E3 $\alpha$, and (ii) full-length DIAP1 is targeted by a process that surprisingly does not require the RING-finger domain and the autoubiquitinating activity of the protein, but rather an exogenous E3, probably DIAP2. Studying the role of the autoubiquitinating activity of DIAP1, we demonstrate that it does not involve internal Lys48 of ubiquitin, but probably Lys63, and the modification appears to attenuate the ligase activity of DIAP1 towards its exogenous substrates.

\section{Results}

Caspase-mediated processing of DIAP1 in vitro. DIAP1 is rapidly degraded upon induction of apoptosis. ${ }^{11,15}$ Recent studies have demonstrated that in the fly, DIAP1 degradation is initiated by limited processing mediated by the caspase DrICE. ${ }^{19,26}$ To further study the mechanism(s) involved and the physiological role of the initial limited processing event in the degradation of DIAP1, we established a stepwise, cell free proteolytic assay. Towards that end, we first incubated $\mathrm{N}$-terminally tagged $6 \times$ Myc $\left[{ }^{35} \mathrm{~S}\right]$ methionine-labeled DIAP1 in HeLa extract and demonstrated that the intact protein was cleaved to generate a slightly shorter C-terminal, $\sim 45 \mathrm{kDa}$ radioactive product (Figure 1a; lanes 2 and 3 ). Since the short, 20 residues $\mathrm{N}$-terminal fragment contains also a $6 \times$ Myc tag, it enabled us to detect this fragment as well (processed DIAP1(S) in Figure 1a). As expected, processing was ATP independent (Figure 1a, lane 2), yet we noted that in the presence of ATP, we were able to detect what appear to be ubiquitin adducts of either the full-length and/or the processed DIAP1 (Figure 1a, lane 3, denoted Conj.). This finding raised the possibility that addition of ATP can target also the intact protein to ubiquitin-mediated degradation without prior processing. It should be noted that the cleavage in HeLa extract was spontaneous (Figures 1a, lane 2 and $1 \mathrm{~b}$, lane 1 ), suggesting that some of the cultured cells contain active caspases, or that they were activated during preparation of the extract. In contrast to the HeLa cells, extracts derived from other mammalian cells could catalyze cleavage only following induction of apoptosis in the intact cells (Figure 1b, lanes 3-6). Analysis of cleaved PARP demonstrated indeed a low spontaneous caspase activity in HeLa cells, in contrast to COS7 and MCF7 cells (not shown).

To further confirm the nature of the enzymatic activity involved in DIAP1 processing, we monitored the reaction in the presence of caspase and proteasome inhibitors. Z-VADFMK is a general caspase inhibitor, and Ac-DEVD-CHO is a potent inhibitor of caspase-3. As can be seen in Figure 1ci, both reagents abolished DIAP1 cleavage. In contrast, MG132 and lactacystin, two potent proteasome inhibitors, did not have any effect (not shown). Strengthening the notion that processing of DIAP1 is mediated by caspase is the finding that DIAP1 mutated in the consensus caspase cleavage site 17-DQVD-20 $\rightarrow$ 17-DQVA-20 (DIAP1 D20A) is resistant to cleavage (Figure 1cii).

Processed DIAP1 is degraded via the ubiquitinproteasome system following recognition of the $\mathrm{N}$-terminal residue ( $\mathrm{N}$-end rule). Ditzel et al. ${ }^{19}$ also demonstrated that the processed C-terminal fragment of DIAP1 is degraded in vivo via the $\mathrm{N}$-end rule pathway, following recognition of its newly exposed destabilizing $\mathrm{N}$-terminal residue (asparagine). To reconstitute in vitro the two-step degradation of DIAP1 - e.g. processing that is followed by complete proteolysis - we first generated processed DIAP1 by incubating ${ }^{35} \mathrm{~S}$-labeled, full-length DIAP1 in the presence of ATP-depleted HeLa cell extract (see above and Figure 1). During the $90 \mathrm{~min}$ incubation period, almost all the protein is processed (Figures 1 and $2 a$, compare lane 1 to lane 2). We then added ATP-containing 
a

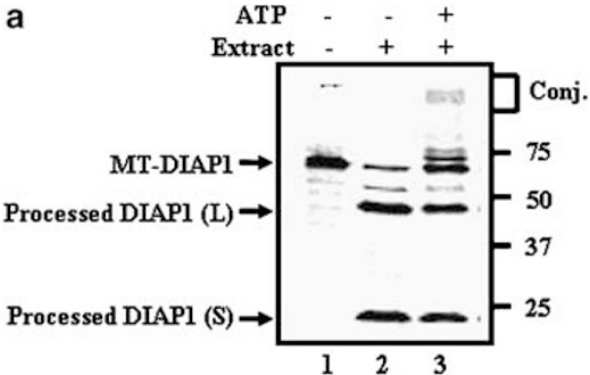

C (i)

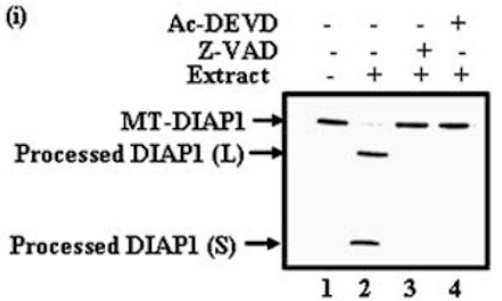

b

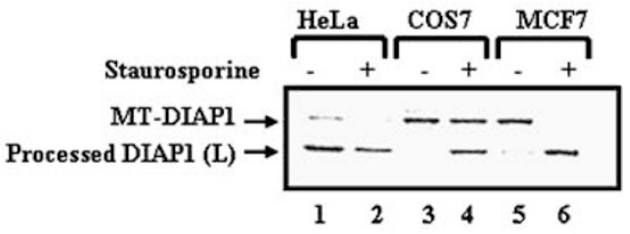

(ii)

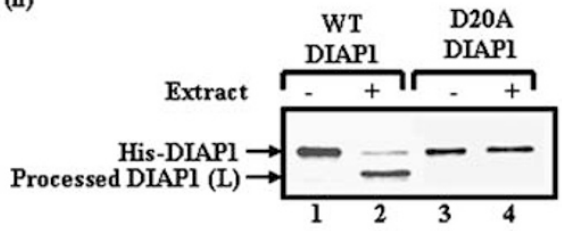

Figure 1 In vitro processing of DIAP1 is caspase dependent. ${ }^{35}$ S]methionine-labeled Myc-tagged panels (a-ci) or His-tagged (panel Cii) DIAP1 were incubated in crude cell extracts, and their processing was monitored as described under 'Materials and Methods'. (a) Processing of DIAP1 is ATP independent. DIAP1 was incubated in the absence (lane 1) or presence (lanes 2 and 3) of HeLa cell extract, and in the absence (lane 2) or presence (lane 3) of ATP. (b) Apoptosis stimulates DIAP1 processing. Apoptosis was induced in cells by staurosporine, and extracts were prepared as described under 'Materials and Methods'. (ci) Effect of caspase inhibitors on processing of DIAP1. Labeled DIAP1 was incubated in the absence (lane 1) or presence (lanes 2-4) of HeLa cell extract, and in the absence (lane 2) or presence of Z-VAD (lane 3) and ACDEVD (lane 4). (cii) Processing of WT DIAP1 requires the specific caspase site 17DQVD20. WT (lanes 1,2) and D20A (lanes 3,4) labeled His-DIAP1 were incubated in the absence (lanes 1,3) or presence (lanes 2, 4) of HeLa cell extract. Proteins were visualized following SDS-PAGE via Phosphorlmaging

reticulocyte lysate that is known to degrade faithfully and efficiently many substrates of the ubiquitin system. As can be seen in Figure $2 a$, addition of the lysate stimulated significantly degradation of processed DIAP1 (compare lane 3 to lane 2). Importantly, degradation of processed DIAP1 requires polyubiquitin chain formation (and therefore also ATP): addition of methylated ubiquitin (MeUb), a polyubiquitin chain terminator, ${ }^{30}$ inhibited degradation almost completely, and the addition of excess WT ubiquitin reversed the inhibition (Figure $2 \mathrm{a}$, compare lane 5 to lane 4). Not surprisingly, addition of MG132 inhibited degradation of the processed fragment in the cell free system (not shown). In order to confirm proteasome involvement in the degradation of DIAP1 in vivo, we transfected MCF7 cells with DIAP1 and followed the fate of the processed fragment. As can be seen in Figure 2b, the processed form could not be detected under basal conditions (lane 1), but appeared following induction of apoptosis by staurosporine (lane 2). Staurosporine-mediated processing of DIAP1 could be completely inhibited by the addition of the caspase inhibitor Z-VAD (lane 3). The accumulation of the processed fragment following addition of MG132 (Figure 2b, lane 4) even in the absence of induction of apoptosis suggests that under basal conditions caspase activity is low, but nevertheless exists, and the processed fragment is rapidly degraded and therefore cannot be detected (Figure $2 b$, lane 1). Following addition of MG132, it is accumulated, and therefore can be detected. Yet, it is also possible that MG132 induces apoptosis, resulting in the formation of processed DIAP1. However it is clear that MG132 also inhibits the degradation of processed DIAP1, as is evident from the experiments described in Figures $2 \mathrm{~d}$ and $2 \mathrm{e}$, where MG132 inhibited the degradation of the processed form that was not generated from caspase cleavage, but rather from constitutive processing of cell-expressed ubiquitin- $\Delta$ N20 DIAP1 fusion chimera.

As noted, Ditzel et al. ${ }^{19}$ demonstrated that the processed fragment is degraded in cells via the $\mathrm{N}$-end rule pathway. Processed DIAP1 contains in its $\mathrm{N}$-terminal position asparagine, a residue that becomes 'destabilizing' following deamidation (to aspartate) and arginylation (see above). As the arginylation reaction requires RNNA $^{\mathrm{Arg}}$, it can be inhibited by the addition of RNase. ${ }^{25}$ Also, the degradation of the already arginylated protein can be specifically inhibited by dipeptides that have a basic residue at their $\mathrm{N}$-terminal position. Dipeptides such as Arg-Ala and Lys-Ala inhibit competitively the degradation of substrates with exposed basic $\mathrm{N}$-termini by binding to the $\mathrm{E} 3 \alpha$ ligase at the substratebinding site that recognizes specifically similar $\mathrm{N}$-termini in the target substrates. The reverse peptides such as Ala-Arg or Ala-Lys are not inhibitory. ${ }^{31}$ In order to demonstrate involvement of E3 $\alpha$ in targeting processed DIAP1 in vitro, we tested the effect of RNAse and the specific inhibitory dipeptides on its degradation. As can be seen in Figure $2 \mathrm{c}$, degradation of processed DIAP1 was completely inhibited by RNAse in a two-step reconstituted cell free proteolytic system (compare lane 4 to lane 3). Prior inhibition of the RNase by RNasin abolished the effect of RNase (Figure 2c, compare lane 5 to lane 4). As expected, addition of Arg-Ala (Figure 2c, lanes 6 and 7), but not of Ala-Arg (lane 8), also inhibited the degradation of the processed fragment of DIAP1. In order to confirm these in vitro results in vivo, we expressed in cells the fusion protein ubiquitin- $\Delta$ N20 DIAP1. Ubiquitin fusion proteins undergo cleavage in cells by constitutive ubiquitin C-terminal proteases that recognize the C-terminal Gly76 of ubiquitin, ${ }^{20}$ resulting in this case in the release of processed DIAP1. As 


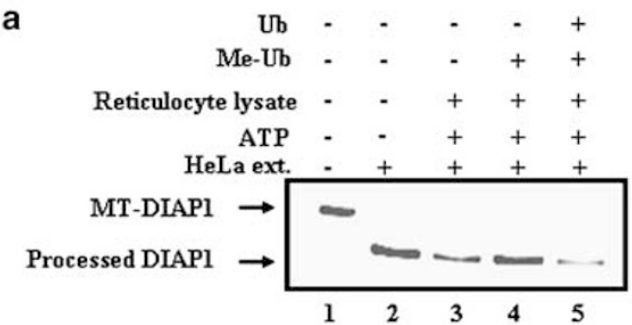

C

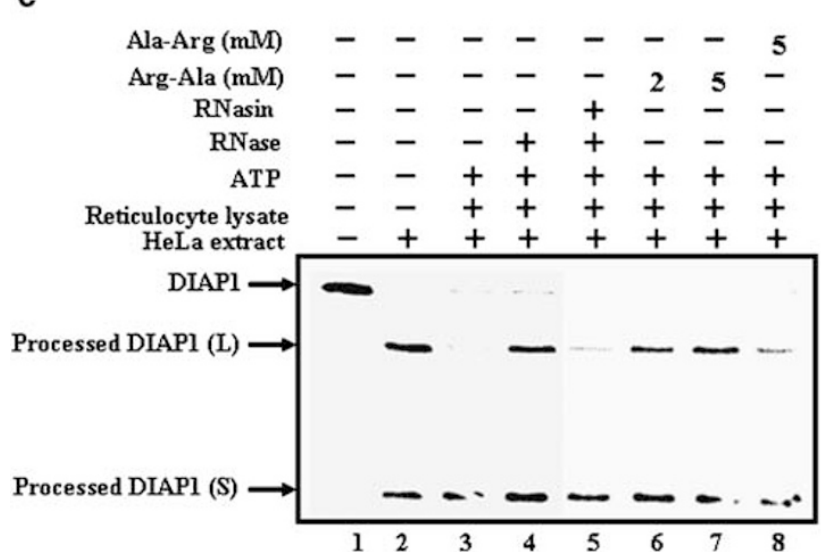

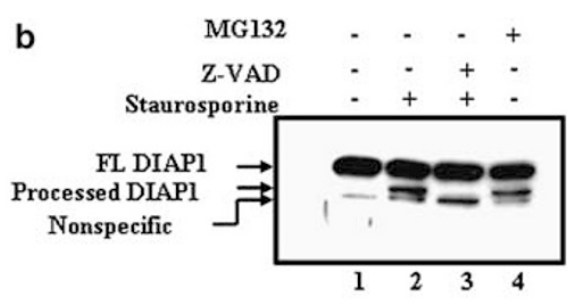

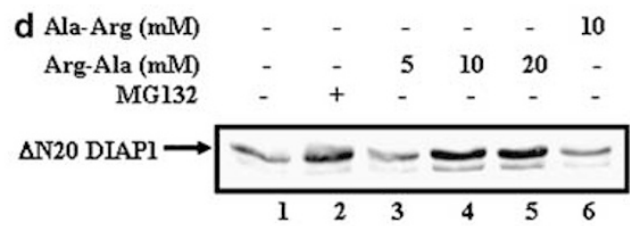

e

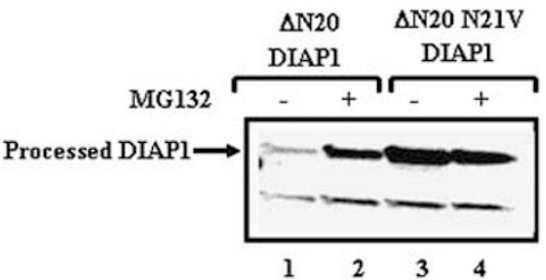

Figure 2 Processed DIAP1 is degraded in vitro and in vivo by the N-end rule pathway. (a) Degradation of processed DIAP1 in a cell free system requires polyubiquitin chain formation. $\left[{ }^{35}\right.$ S $]$ methionine-labeled MT-DIAP1 was incubated in the absence (lane 1) or presence (lanes 2-5) of HeLa cell extract, followed by the addition of reticulocyte lysate as indicated and described under 'Materials and Methods'. ATP, MeUb and Ub were added as indicated. Proteins were resolved via SDS-PAGE and visualized by Phosphorlmaging. (b) Degradation of processed DIAP1 in cells is proteasome dependent. MCF7 cells were transiently transfected with cDNA coding for FL DIAP1 as described under 'Materials and Methods'. Staurosporin (lanes 2 and 3), Z-VAD (lane 3) and MG132 (lane 4) were added, and the fate of DIAP1 was monitored using Western blot analysis as described under 'Materials and Methods'. (c) Degradation of processed DIAP1 in a cell free system is sensitive to RNase and to the dipeptide Arg-Ala. $\left[{ }^{35}\right.$ S]methionine-labeled MT-DIAP1 was subjected to in vitro processing (by HeLa cell extract) and subsequent degradation (by the addition of reticulocyte lysate) as described under 'Materials and Methods' and indicated in the figure. Lane 1, labeled DIAP1. Lane 2, processed DIAP1 generated by HeLa cell extract. Lane 3-8, degradation of processed DIAP1 is mediated via the N-end rule. Lane 3, degradation of processed DIAP1 following the addition of reticulocyte lysate and ATP. Lane 4, as lane 3, but in the presence of $0.15 \mu \mathrm{g}$ RNase A. Lane 5, as lane 4, but RNasin (60 units) was added 5 min prior to the addition of RNase. Lanes 6 and 7 , effect of Arg-Ala (2 and $5 \mathrm{mM}$, respectively) on the degradation of processed DIAP1. Lane 8 , effect of Ala- $\operatorname{Arg}(5 \mathrm{mM})$ on the degradation of processed DIAP1. Bestatin was present in all assays ( $25 \mu \mathrm{g} / \mathrm{ml})$ to inhibit proteolysis of the dipeptides. (d) Effect of Arg-Ala and Ala-Arg on the degradation processed DIAP1 in cells. MCF7 cells were transiently transfected with cDNA coding for ubiquitin- $\triangle 20$ DIAP fusion protein. The fate of processed DIAP1 was monitored following addition of MG132 (lane 2) and the indicated concentrations of Arg-Ala (lanes 3-5) or Ala-Arg (lane 6) to the growth medium $6 \mathrm{~h}$ prior to harvesting the cells. The processed protein was detected using anti-FLAG antibody as described under 'Materials and Methods'. Bestatin was added to the medium at a final concentration of $2.5 \mu \mathrm{g} / \mathrm{ml}$. (e) Degradation of processed DIAP1 in cells is dependent on the presence of a destabilizing $N$-terminal residue. S2 cells were transiently transfected with cDNAs coding for ubiquitin- $\triangle N 20$ DIAP1 (lanes 1,2), or ubiquitin- $\triangle$ N20 N21V DIAP1 (lanes 3,4). MG132 was added as indicated, and the fate of the ubiquitin hydrolase-processed DIAP1 was followed via Western blot analysis as described under 'Materials and Methods'

can be seen in Figure $2 d$ and similar to the results obtained in the cell free system, Arg-Ala (lanes 3-5), but not Ala-Arg (lane $6)$, inhibited the degradation of the processed fragment when added to the cell growth medium. Furthermore, when the asparagine residue at position 21 was substituted with valine, a 'stabilizing' residue, ${ }^{20}$ the processed DIAP1 was stable (Figure 2e, compare lane 3 to lane 1). Taken together, these data support the notion that processed DIAP1 is degraded by $\mathrm{E} 3 \alpha$ following recognition of the newly exposed $\mathrm{N}$-terminal asparagine, and its two-step - deamidation and arginylation - conversion to arginine.

Bachmair and Varshavsky ${ }^{32}$ demonstrated that besides the destabilizing $\mathrm{N}$-terminal residue, there are one or two specific lysine residues at the $\mathrm{N}$-terminal domain that function as a second determinant in the degradation signal, serving as ubiquitin acceptors in the model protein they studied. Ditzel et al. ${ }^{19}$ suggested, but have not shown, that lysine residues 10 and 15 in processed DIAP1 (residues 30 and 35 in the full-length protein) may serve as ubiquitin anchors in this protein. We replaced these two residues with arginine (K30,35R DIAP1) and obtained a processed fragment that was still unstable, ruling out a role for these two lysine residues as ubiquitin acceptors (not shown).

Full-length, non-processable DIAP1 is degraded by the ubiquitin system in a mechanism that does not require its RING-finger domain. While Yokokura et al. ${ }^{26}$ have recently reported that the cleavage of DIAP1 promotes its degradation in a manner that is dependent on the protein's RING-finger domain, the findings of Ditzel et al. ${ }^{19}$ and our own (see above) argue that the processed form is degraded via the $\mathrm{N}$-end rule pathway, using $\mathrm{E} 3 \alpha$ as the modifying ligase. However, the problems of the stability of intact DIAP1 and whether caspase-mediated processing is essential for degradation of the protein have remained unsolved. To solve these questions, we monitored in Drosophila S2 cells the 
half-life of endogenous DIAP1 in the presence and absence of the caspase inhibitor Z-VAD. As can be seen in Figure $3 a$ (lanes 1-4), intact DIAP1 is degraded rapidly $\left(t_{1 / 2}\right.$ of $\sim 40 \mathrm{~min})$, and its stability is independent on caspase activity (lanes 5-8), suggesting that the enzyme can be degraded via an additional, caspase and $\mathrm{N}$-end ruleindependent pathway. As the paradigm in the ubiquitin system research area is that RING finger domain ligases are involved in their own destruction via autoubiquitination, it was logical to assume that this is also the case with DIAP1. To test this hypothesis, it was important to inactivate DIAP1 autoubiquitinating activity. Accordingly, we mutated a critical Ile residue in the RING finger domain of the protein (DIAP1 I393A) that was reported to inactivate the autoubiquitinating activity of other ligases without affecting their RING finger structure. $^{33}$ As can be seen in Figure $3 b$, the mutation abolished the self-conjugating activity of the enzyme even in the presence of Reaper, the strong proapoptotic protein. Surprisingly, similar to the WT protein, I393A DIAP1 was also unstable in cells (Figure 3ci, compare lanes 4-6 to lanes 1-3). To ascertain that the instability is not due to prior caspase-mediated limited cleavage, we monitored the stability of the nonprocessable D20A DIAP1 and found that it is similarly unstable (lanes 7-9). Needless to say that the double mutant I393A,D20A was also unstable (lanes 10-12), and its degradation was proteasome-dependent (Figure 3cii). Assuming that other, yet to be discovered cleavages can also lead to subsequent degradation of the cleaved product (see for example, Muro et al. ${ }^{34}$ ), it was important to test whether the RING finger domain can be involved in the degradation of a

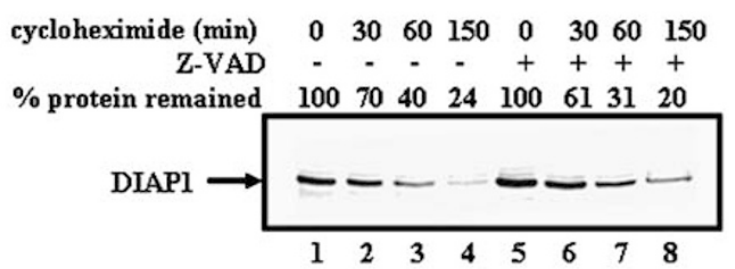

b

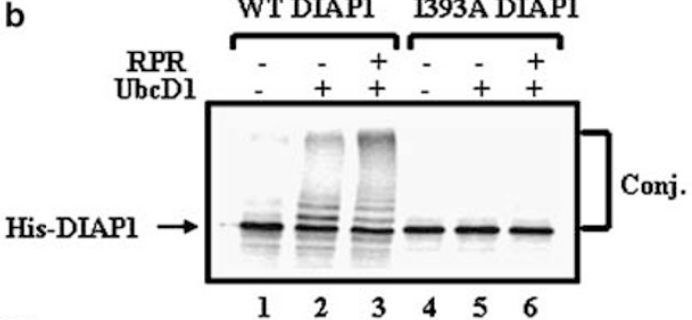

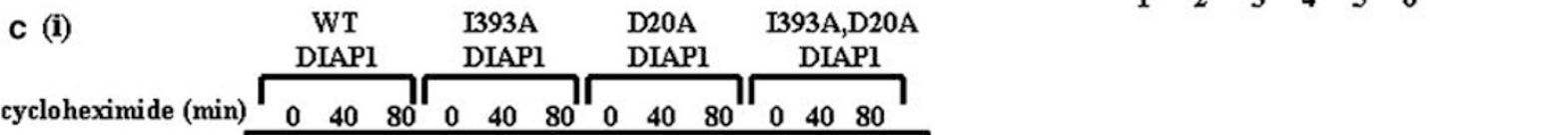

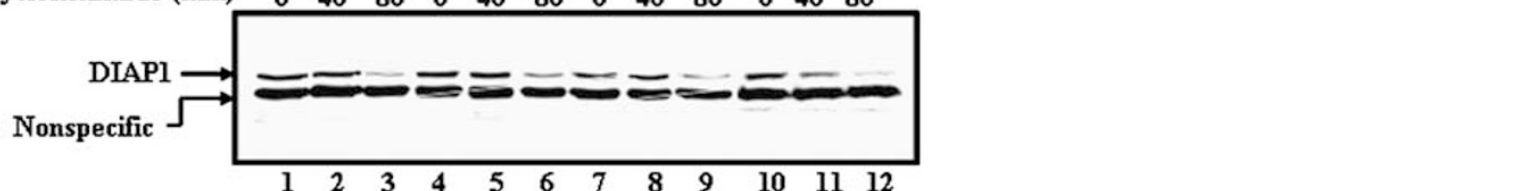

(ii)

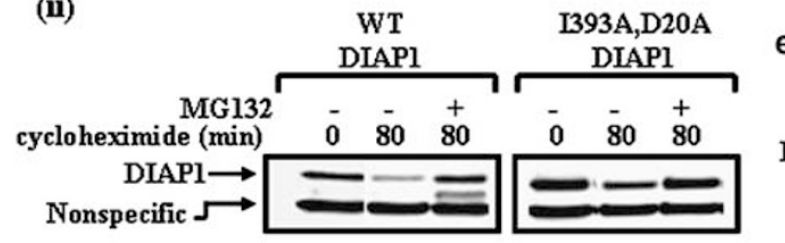

e (i)

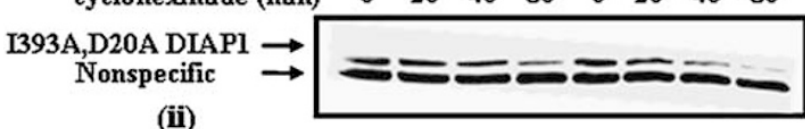

d

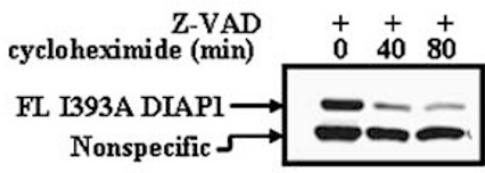

(ii)

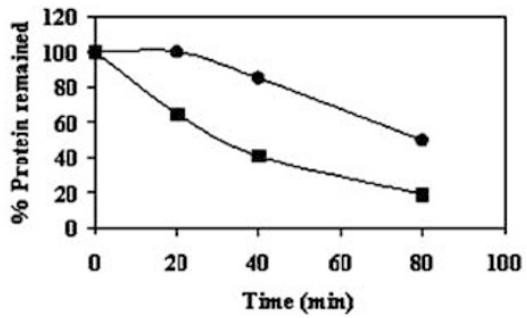

Figure 3 Processing of DIAP1 is not essential for its degradation and does not require its RING finger domain. (a) DIAP1 is rapidly degraded in the presence or absence of caspase inhibitor. S2 cells were incubated in the absence or presence of Z-VAD, and the stability of endogenous DIAP1 was monitored following the addition of cycloheximide for the indicated times as described under 'Materials and Methods'. (b) DIAP1 requires its RING domain for autoubiquitination in vitro. ${ }^{35}$ S]methionine-labeled WT (lanes 1-3) and I393A DIAP1 (lanes 4-6) were subjected to in vitro self ubiquitination as described under 'Materials and Methods'. (c) (i) DIAP1 proteins mutated in the RING-finger domain and the caspase cleavage site are unstable. cDNAs coding for WT, I393A,D20A, and I393A,D20A DIAP1s were transfected into S2 cells, and the stability of the proteins was monitored following the addition of cycloheximide as described under 'Materials and Methods'. (ii) DIAP1 mutated in both the RING-finger domain and the caspase cleavage site is degraded in a proteasome-dependent manner. CDNAs coding for WT and I393A,D20A DIAP1 were transfected into S2 cells, and the fate of the proteins was followed in the absence or presence of MG132 as indicated and described under 'Materials and Methods'. (d) RING-finger domain-mutated DIAP1 is degraded in the absence of caspase activity. CDNA coding for I393A DIAP1 was transfected into S2 cells, and the stability of the expressed protein was measured following the addition of cycloheximide in the presence of Z-VAD as described under 'Materials and Methods'. (e) (i) Degradation of I393A,D20A DIAP1 is stimulated following induction of apoptosis. cDNA coding for I393A,D20A DIAP1 was transfected into S2 cells. Etoposide was added for $8 \mathrm{~h}$, and the stability of the proteins was monitored following the addition of cycloheximide as described under 'Materials and Methods'. (ii) Quantification of the results shown in (i); $\bullet$, without etoposide, $\mathbf{\square}$, with etoposide 
an intact protein that cannot be cleaved by any caspase. As can be seen in Figure 3d, Z-VAD does not have an effect on the degradation of I393A DIAP1, suggesting that the intact protein is degraded without any prior cleavage in a mechanism that does not require its RING finger domain and autoubiquitination. Degradation of the protein must therefore be mediated by an exogenous E3 that is distinct from E3 $\alpha$ (see below).

All these experiments were carried out under basal conditions. At that point, it was important to study the effect of apoptosis on the degradation of the RING finger and caspase site double mutant DIAP1. That in order to test whether under apoptotic conditions intact DIAP1 can be rapidly degraded, thus removing antiapoptotic activity and enabling the programmed cell death machinery to proceed efficiently. As can be seen in Figure 3e, addition of etoposide resulted in an $\sim 2-3$-fold acceleration in the degradation of the RING finger and caspase site double mutant DIAP1. This finding suggests that induction of apoptosis stimulates degradation of DIAP1 via a novel, ubiquitin- and proteasomemediated mechanism that does not require prior processing or self destruction of the enzyme, and is probably independent of the $\mathrm{N}$-end rule pathway.

DIAP1 generates self Lys63-based polyubiquitin chains that appear to regulate its activity rather then targeting it for degradation. As autoubiquitination of DIAP1 does not lead to its degradation, we suspected that the polyubiquitin chains formed are not of the 'canonical' Lys48-based type. It was therefore important to determine the identity of the lysine residue(s) of ubiquitin involved in their formation. Towards that end, we used ubiquitin mutants in which all but one (indicated by number) lysine residue were substituted with arginine (K6, K11, K27, K29, K33, K48, and K63 ubiquitins). As can be seen in Figure 4a, ubiquitin K63 was the most efficient species in catalyzing polyubiquitination, although it was less efficient than the WT molecule (compare lane 7 to lane 1). Importantly, K48 was not able to generate high molecular mass adducts, although it was somewhat more efficient than lysine-less ubiquitin (K0; compare lane 6 to lane 8). DIAP1 could use also K11 ubiquitin, but not as efficiently as K63 (compare lane 3 to lanes 7 and 8 ). These data suggest that UbcD1 can catalyze formation of Lys63-based polyubiquitin chains on DIAP1. Ubc13 is the E2 that was shown, as part of a complex with another partner, Uev1a, to be involved in many cases of assembly of such chains. ${ }^{35,36}$ However, several cases were described recently in which 'canonical' E2s were also shown to be involved in generation of such chains. ${ }^{37,38}$ While a Ubc13 homolog was described also in the fly, ${ }^{39}$ it appears from our in vitro data that a 'canonical' E2 such as UbcD1 can also be involved in the process. We nevertheless decided to examine the role of Ubc13/Uev1a in DIAP1 autoubiquitination in vitro. As can be seen in Figure 4b, the Ubc13/Uev1a complex can catalyze polyubiquitin chain formation on DIAP1 using K63 ubiquitin (lane 4), although at a lower efficiency compared to the chains generated with WT (lane 1) or K48R (lane 5) ubiquitins (that harbor intact Lys63 residue). The low efficiency can be due to the fact that additional lysines are required to generate a mixed, although K63-containing chain.
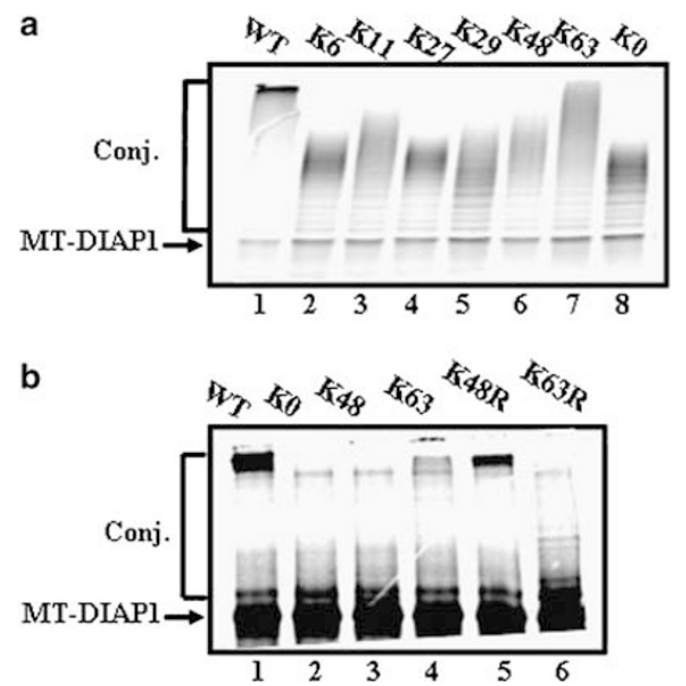

Figure 4 DIAP1 autoubiquitination generates mostly K63-based polyubiquitin chains. (a) K63 ubiquitin demonstrates the highest efficiency in DIAP autoubiquitination. Myc antibody-immobilized $\left[{ }^{35}\right.$ S]methionine-labeled MT-DIAP1 was subjected to in vitro self ubiquitination in the presence of $\mathrm{UbcD1}$ (a) or Ubc13/ Uev1a (b) as described under 'Materials and Methods'. WT, single lysine-containing ubiquitin proteins ( $\mathrm{K}$ mutants, where $\mathrm{Kn}$ indicates the position of the single lysine residue remained in the ubiquitin molecule) or $\mathrm{K}>\mathrm{R}$ ubiquitin mutants were added as indicated. Conj. denotes conjugates

Also, it is possible that the multiply mutated $\mathrm{K} 63$ ubiquitin in which six lysine residues were altered is folded in such a conformation that is less efficiently recognized by the Ubc13/ Uev1a conjugating complex and by DIAPI.

Self-ubiquitination of DIAP1 is probably involved in regulating the enzyme's activity. From the instability of the RING finger mutant DIAP1 and the nature of its selfgenerated polyubiquitin chains, we hypothesized that they probably serve a nonproteolytic function, such as regulating the enzyme's activity. Thus, we devised a two-step ubiquitination reaction in which we tested DIAP1 activity towards its substrate Dronc before and after selfubiquitination: (i) first, we generated ubiquitinated DIAP1 by incubating the purified enzyme in the presence or absence of ubiquitin (Figure 5a). (ii) Next, we added to each reaction mixture ${ }^{35}$ S-labeled Dronc and WT ubiquitin, and followed Dronc ubiquitination. As can be seen in Figure 5b, if Dronc was added simultaneously with all reaction components, it was ubiquitinated much more efficiently compared to when added following DIAP1 ubiquitination (Figure 5b, compare lane 2 to lane 3). This experiment suggests that the polyubiquitin chain can serve to attenuate the ligase activity of DIAP1 towards its substrate. Further corroborating this notion is the experiment described in Figures $5 \mathrm{c}$ and $5 \mathrm{~d}$. Here we used lysine-less (KO) ubiquitin that cannot polymerize. As expected, utilization of this ubiquitin resulted in significantly lower molecular mass adducts of DIAP1 compared to those generated when WT ubiquitin was used (Figure 5c, compare lane 3 to lane 2; see also Figure 4a, lane 8 ), reflecting probably single ubiquitination on multiple distinct lysine residues. Yet, despite modifying lysine residues only once, the majority of adducts of Dronc 
a

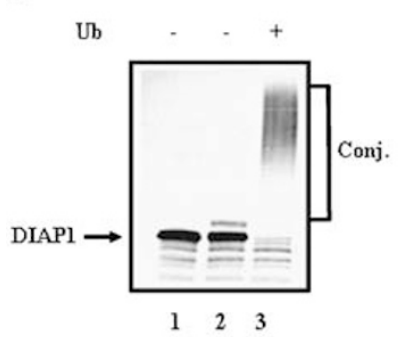

C

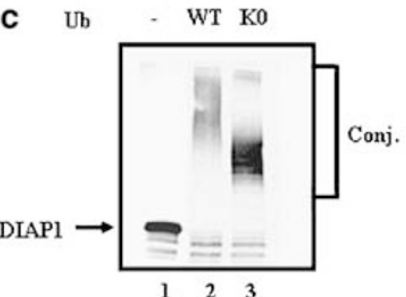

b

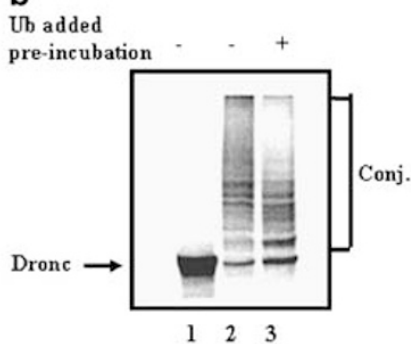

d

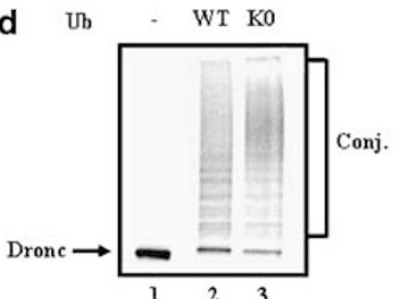

Figure 5 Self-ubiquitination of DIAP1 is involved in attenuating the ligase activity of the enzyme towards an exogenous substrate. (a) Bacterially expressed GST-DIAP1 was subjected to in vitro self ubiquitination in the absence (lane 2) or presence (lane 3) of WT ubiquitin. Conjugated DIAP1 was detected following SDSPAGE via Western blot analysis as described under 'Materials and Methods'. (b) DIAP1 was preincubated for $30 \mathrm{~min}$ in the absence or presence of ubiquitin as described under (a). After the preincubation, ${ }^{35}$ S $]$ methionine-labeled Dronc and ubiquitin were added, and conjugated Dronc was visualized following SDS-PAGE and Phosphorlmaging as described under 'Materials and Methods'. (c, d) Bacterially expressed GST-DIAP1 and $\left[{ }^{35}\right.$ S $]$ methionine-labeled Dronc were subjected to in vitro ubiquitination with WT or KO ubiquitin as indicated and described under 'Materials and Methods'. Lanes 1 in all panels represent the amount of the substrate protein added to the reaction mixture (no incubation). Conj. denotes conjugates

generated using $\mathrm{KO}$ ubiquitin were of higher molecular mass compared to those formed using WT ubiquitin (Figure $5 \mathrm{~d}$, compare lane 3 to lane 2. Compare also Figure $5 \mathrm{~d}$ lane 3 to Figure $5 \mathrm{~b}$ lane 3 ). Supporting the higher efficiency of ubiquitination in the presence of $\mathrm{KO}$ ubiquitin is the corollary larger reduction in the amount of free, unconjugated Dronc remained in the mixture (Figure $5 \mathrm{~d}$, compare the free Dronc in lanes 3 and 2, respectively). We hypothesize that the polymerized ubiquitin chains attenuate DIAP1 activity, possibly by steric hindrance, which the single modifications do not.

DIAP2 binds DIAP1, ubiquitinates it and regulates its stability. Silke et al. ${ }^{40}$ showed that mammalian clAP1 binds directly to XIAP, leading to its ubiquitination and subsequent proteasomal degradation. As our data suggest that DIAP1 autoubiquitination is not involved in its targeting, we tested the possibility that a similar mechanism operates also in Drosophila, in which DIAP2 regulates DIAP1. As can be seen in Figure 6a, DIAP2 binds to DIAP1 (lanes 2 and 3), and the binding is specific: GST and GST-Max, unlike GST-DIAP1, do not bind DIAP2 (lanes 4-6). Furthermore, our data demonstrate that DIAP2 ubiquitinates RING finger mutant DIAP1 in a cell free conjugation assay (Figure 6bi, lanes 3 and 4). The utilization of the self ubiquitination-defective DIAP1 enabled us to detect only the exogenous DIAP2 activity without the background self activity.
To examine whether DIAP2 is involved in DIAP1 degradation, we first tested the structure of the polyubiquitin chains formed in the cell free ubiquitination assay. We used two ubiquitin mutants that harbor K48 (K48 and K63R), and two that do not have this lysine residue (K48R and K63; the $\mathrm{K}$ mutants have only the indicated lysine residue left, while the $\mathrm{K}>\mathrm{R}$ mutants have the indicated lysine substituted with $\mathrm{R}$ ). As can be seen in Figure 6bii, DIAP2 could catalyze generation of high molecular mass polyubiquitin chains on DIAP1 only when ubiquitins K48 and K63R were present. These findings support the notion that the chains synthesized are Lys48-based, leading most probably to the degradation of DIAP1. Additional experiments carried out using S2 cells further support the notion that DIAP2 is involved in DIAP1 degradation following its ubiquitination: (i) silencing of DIAP2 expression results in accumulation of DIAP1 (Figure 6ci; compare lanes 1 and 2 to lanes 3-6). (ii) DIAP1 was significantly more stable in the presence of DIAP2 RNAi than in the presence of GFP RNAi (Figure 6cii). (iii) Expression of RING finger mutant, dominant negative species of DIAP2 (V453A), resulted in significant stabilization of DIAP1 (Figure 6ciii).

\section{Discussion}

It was reported ${ }^{19}$ that caspase-catalyzed processing of DIAP1 and subsequent $\mathrm{N}$-end rule-mediated degradation of the $\mathrm{C}$ terminally released fragment are essential for the destruction of the enzyme. Ubiquitination of $\mathrm{N}$-end rule substrates is mediated by $\mathrm{E} 3 \alpha$ that binds to its target substrates via their destabilizing $\mathrm{N}$-terminal residues. DIAP1 is a RING finger domain-containing ubiquitin ligase that catalyzes its own ubiquitination, a reaction believed to target RING finger E3s for degradation. Thus, at first glance it appears that DIAP1 can be degraded via two distinct ubiquitination pathways: (i) initial caspase-dependent processing and $\mathrm{N}$-end rule/E3 $\alpha$ mediated degradation of the processed fragment, and (ii) RING finger-mediated autoubiquitination of the full-length protein or of the processed fragment. We set to study the role of these pathways in governing DIAP1 stability.

Initially, we reconstituted a cell free system that faithfully reproduces the degradation of DIAP1 and demonstrated that, as reported, it can proceed in two steps: (i) processing of the intact protein, and (ii) degradation of the released fragment (Figures 1 and 2). We also showed that in both the cell free system and cells, the processed fragment is degraded by the ubiquitin system via the $\mathrm{N}$-end rule pathway (Figure 2).

At that point it was important to study whether the initial processing is essential for degradation of the enzyme, or whether the intact enzyme can also be degraded. As can be seen in Figure 3a, inhibition of caspase activity by Z-VAD had no effect on the stability of DIAP1.

As $\mathrm{N}$-end rule-mediated ubiquitination of the processed fragment is catalyzed by an exogenous ligase, E3 $\alpha$, we wanted to study whether the RING finger-mediated autoubiquitinating activity of DIAP1 is also involved in governing its stability. It should be noted that following processing, the RING finger in the processed fragment remains intact. Surprisingly, a mutation in the RING finger domain that inactivates the autoubiquitinating activity had no effect on the 


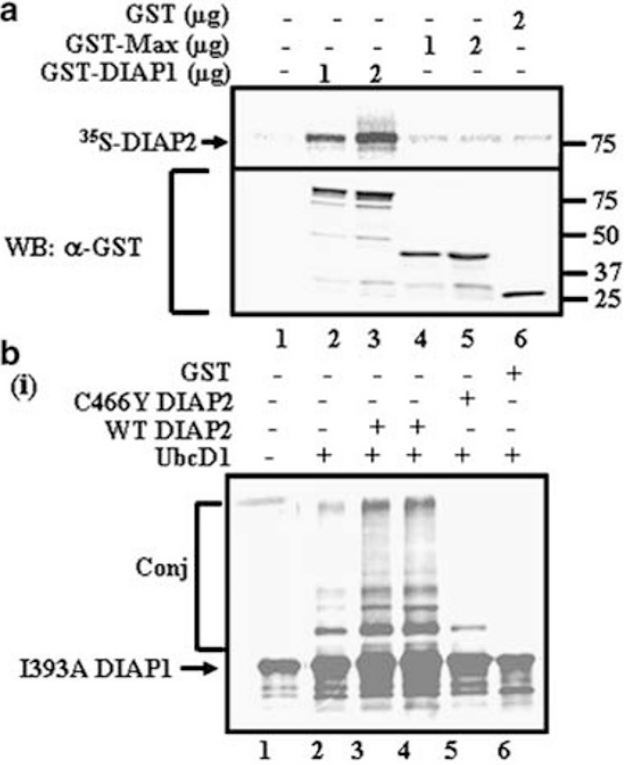

(ii)

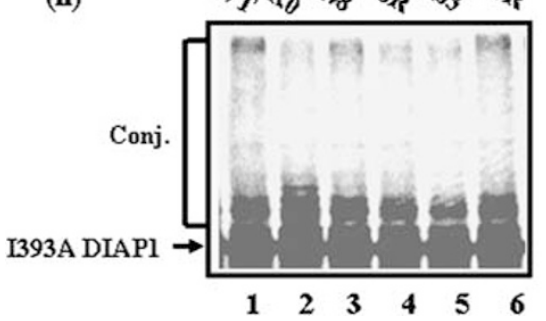

c

(i)

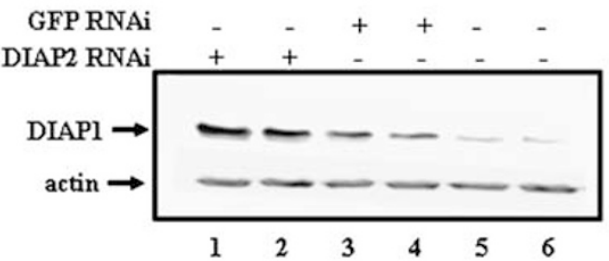

(ii)

$\begin{array}{rcccc}\text { GFP RNAi } & - & - & + & + \\ \text { DIAP2 RNAi } & + & + & - & - \\ \text { eximide (min) } & 0 & 80 & 0 & 80\end{array}$
cycloheximide (min)

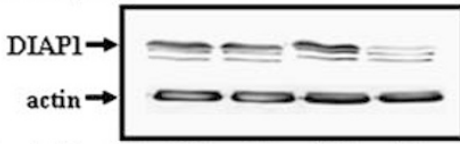

DIAP1 (\% relative to time 0$) \quad 100 \quad 65 \quad 100 \quad 14$

(iii)

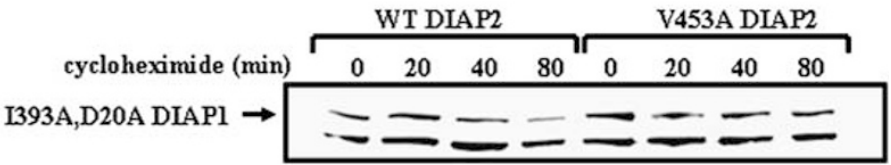

Figure 6 DIAP2 binds DIAP1 in a specific manner (a), ubiquitinates it (b), and is involved in its degradation (c). (a) DIAP1 binds DIAP2 in a specific manner. In vitro translated and ${ }^{35}$ S]methionine-labeled DIAP2 was incubated in the presence of the indicated amounts of bacterially expressed GST-DIAP1 (lanes 2 and 3), GST-Max (lanes 4 and 5) and GST (lane 6). Following incubation for $30 \mathrm{~min}$ at $25^{\circ} \mathrm{C}$, the reaction mixtures were immobilized to glutathione-agarose as described under 'Materials and Methods'. Upper panel, autoradiogram of the labeled DIAP2. Lower panel, Western blot analysis of the various GST-containing proteins using anti-GST antibody. (bi) Ubiquitination of DIAP1 by DIAP2. [ ${ }^{35}$ S]methionine-labeled I393A DIAP1 was subjected to in vitro ubiquitination in the presence or absence of bacterially expressed purified GST-DIAP2 or GST as described under 'Materials and Methods'. (bii) DIAP2 catalyzes formation of Lys48-based polyubiquitin chains on DIAP1. ${ }^{35}$ S]methionine-labeled I393A DIAP1 was subjected to in vitro ubiquitination in the presence of UbcD1 and purified DIAP2 as described under 'Materials and Methods'. WT and the different ubiquitin lysine mutants were added as indicated. $\mathrm{K}$ indicates the position of the single lysine residue remained in the ubiquitin molecule, while $\mathrm{KnR}$ indicates the position of the lysine residue substituted with arginine. Conj. denotes conjugates. (c) DIAP2 regulates DIAP1 level in cells. DIAP2 RNAi was prepared as described under 'Materials and Methods' and was added to S2 cells for $60 \mathrm{~h}$. Levels (ci) and stability of endogenous DIAP1 (cii) were monitored as described under 'Materials and Methods'. (ciii) cDNA coding for WT or V453A RING mutant dominant negative DIAP2 were transfected into S2 cells, and the stability of the RING finger and caspase site mutant transfected. DIAP1 was monitored following the addition of cycloheximide as described under 'Materials and Methods'

degradation of the intact protein (Figure $3 c$ and see text) and the processed fragment (not shown). While one can argue that the RING finger mutant can still undergo processing prior to $\mathrm{N}$-end rule-mediated degradation of the cleaved fragment, we have shown that the RING-finger domain was not necessary even for the degradation of a caspase site mutant DIAP1 that cannot be processed (Figures $3 c$ and $3 d$ ). Taken together, these findings imply that the intact protein is targeted by an exogenous, yet to be identified ubiquitin ligase. It was previously shown that expression of Rpr, which leads to disappearance of WT DIAP1 in Drosophila embryos, does not result in disappearance of RING finger mutant DIAP1. These findings raised the hypothesis that Rpr-stimulated and RING finger-mediated autoubiquitination targets the protein for accelerated degradation. ${ }^{9}$ It is possible that Rpr-induced autoubiquitination generates different polyubiquitin chains compared to those formed by the ligase in the absence of Rpr.
These chains should be of the Lys48-based type, recognized by the $26 \mathrm{~S}$ proteasome, while the chains generated by DIAP1 in the absence of Rpr probably use different lysine residues in the ubiquitin moieties (see above and below), and possibly also different lysine residues within DIAP1 itself. Alternatively, in the DIAP1 mutant used in the embryos, ${ }^{9}$ the structure of the RING had been perturbed, and consequently the exogenous E3 that targets the protein cannot bind to it anymore, resulting in its stabilization. Identification of the E3 of DIAP1 and characterization of its binding characteristics should resolve this enigma (see above and below).

In light of the finding that the autoubiquitinating activity of DIAP1 is not required for its targeting, it was important to analyze the structure of the polyubiquitin chain(s) generated by the enzyme. Surprisingly, our findings (Figure 4) suggest that the 'canonical' Lys48-based chain that targets substrates for proteasomal degradation is not involved in the 
autoubiquitinating activity of DIAP1. Rather, it appears that the self chains generated are mostly of the Lys63-based type. An interesting question relates to the nonproteolytic function of the autoubiquitinating activity of DIAP1. One possibility is that it activates the enzyme, similar to the activation of the IKK kinase complex ${ }^{41}$ by Lys63-based polyubiquitin chains. Alternatively, it can attenuate the enzyme's activity. Our initial findings suggest that the self ubiquitination attenuates the activity of the enzyme towards one of its native cellular substrate, the initiator caspase Dronc (Figure 5). We have shown that prior autoubiquitination of DIAP1 inhibits the activity of the enzyme and results in generation of significantly lower molecular mass adducts on Dronc compared to those generated when free, initially unconjugated DIAP1 was added to the reaction mixture (Figure $5 \mathrm{~b}$ ). Furthermore, when lysineless, K0 ubiquitin was used, DIAP1 was more active compared to when WT ubiquitin was used (Figures $5 \mathrm{c}$ and $5 \mathrm{~d})$. We suggest that the multiple modifications of DIAP1 by single ubiquitin moieties do not inhibit the enzyme as the polymerized ubiquitin chains do.

For XIAP1, it has been shown the clAP1 targets it for degradation. ${ }^{40}$ As we demonstrated that the self ubiquitinating activity of DIAP1 does not target it for degradation, we tested the hypothesis that DIAP2 is involved in the process. Four lines of experimental evidence presented in Figure 6 support this notion: (i) DIAP2 binds to DIAP1 specifically and (ii) catalyzes its ubiquitination, generating Lys48-based polyubiquitin chains that are known to target substrates for proteasomal degradation. Also, (iii) silencing of DIAP2 or (iv) expressing a dominant negative species of the enzyme in cells stabilize DIAP1. Such an internal regulatory loop, where one IAP regulates the other, can ensure fine tuning of the activity of these group of proteins following different stimuli and under varying pathophysiological conditions, although the mechanistic details of this regulatory loop are still elusive. Also, this novel mechanism further strengthens the notion that autoubiquitination does not necessarily, as has been thought thus far, leads to self destruction of the involved ligases that are targeted by an exogenous ligase. It should be noted that a role has been suggested for Morgue in promoting the degradation of DIAP1, although it has not been shown that it serves as its ligase. ${ }^{42,43}$ Also, a recent study demonstrates that phosphorylation of DIAP1 by the Drosophila IKK-related kinase leads to its destruction under nonapoptotic conditions. ${ }^{44}$ Thus, it appears that multiple pathways and mechanisms regulate the IAP group of proteins that are positioned in a critical cross roads along the developmental and apoptotic pathways.

\section{Materials and Methods}

Materials. Materials for SDS-PAGE, Bradford protein determination reagent, and prestained molecular weight markers were from Bio-Rad. A mixture of ${ }^{35} \mathrm{~S}$ labeled methionine and cysteine for metabolic labeling, and $\left[{ }^{35} \mathrm{~S}\right]$ methionine for in vitro translation were obtained from GE Healthcare. For site-directed mutagenesis, we used the QuickChange ${ }^{\mathrm{TM}}$ kit from Stratagene. dsRNA for RNA silencing was prepared using the MEGAscript RNA kit (Ambion). Tissue culture sera and media were from Biological Industries (Bet Haemek, Israel), or from Sigma. Anti-DIAP1 was raised in rabbits as described previously. ${ }^{9}$ Mouse anti-Myc tag antibody (9E10) was from Santa Cruz, mouse anti-FLAG was from Sigma, and peroxidaseconjugated goat anti-rabbit or donkey anti-mouse antibodies were from Jackson ImmunoResearch Laboratories. Anti-Poly (ADP-Ribose) Polymerase (PARP) was from Serotec. Ubiquitin, dithiothreitol (DTT), adenosine-5'-triphosphate (ATP), phosphocreatine, creatine phosphokinase, 2-deoxyglucose, glutathione, glutathione immobilized to agarose beads, isopropyl $\beta$-D-thiogalactopyranoside (IPTG), deoxycholic acid, Tris buffer (Trizma base; Tris(hydroxymethyl)aminometane HCL), RNase, RNasin, Arg-Ala, Ala-Arg, etoposide and staurosporine were from Sigma. Ni-agarose was from Qiagene. $\mathrm{N}$-CarbobenzoxyL-leucyl-L-leucyl-leucinal (MG132), Ac-DEVD-CHO, N-BenzyloxycarbonylVal-Ala-Asp (O-Me) fluoromethyl ketone (Z-VAD-FMK), lactacystin $\beta$-lactone, $N$-(2-Hydroxymethyl)piperazine- $N$-(2-ethanesulfonic acid) (HEPES) buffer, and protease inhibitors mixture were from Calbiochem. Immobilized protein $\mathrm{G}$, Adenosine- $5^{\prime}-\left(\gamma\right.$-thio)triphosphate $($ ATP $\gamma S)$, hexokinase and Fugene6 ${ }^{\text {TM }}$ transfection reagent were from Roche Molecular Biochemicals. Ubiquitin aldehyde (UbAl) and methylated ubiquitin (MeUb) were from BIOMOL Research Laboratories. Ubc13/Uev1a complex was from Boston Biochemicals. Reagents for enhanced chemiluminescence (ECL) were from Pierce. TNT ${ }^{\mathrm{TM}}$ Quick coupled transcription-translation kit for in vitro protein synthesis was from Promega. Restriction and modifying enzymes were from New England Biolabs. Oligonucleotides were synthesized by Sigma. All other reagents were of high analytical grade.

Vectors and protein expression. cDNA coding for DIAP1 was subcloned into the MT-pCS2 + vector in frame with N-terminal $6 \times$ Myc tag and downstream to CMV and SP6 promoters (MT-DIAP1). N-terminal $6 \times$ His-tagged DIAP1 (HisDIAP1) subcloned into the pET14b vector (Novagen) was expressed in Escherichia coli and translated in vitro in the presence of $\left.{ }^{35} \mathrm{~S}\right]$ methionine. D20A,I393A and D20A,I393A DIAP1 mutants were generated via site-directed mutagenesis using the His-DIAP1-coding plasmid. For transient transfection in MCF7 cells, DIAP1 and its various mutants were subcloned into the PCMV 5 mammalian expression vector (Sigma). To express the N-terminally processed species of DIAP1 ( $\triangle$ N20 DIAP1, $\triangle N 20$ I393A, and $\triangle N 20 \mathrm{~N} 21 \mathrm{~V}$ ), the corresponding CDNAs were subcloned downstream to the ORF of ubiquitin, using the pCDNA3 vector. For expression in Drosophila S2 cells, DIAP1 and its various mutants and fusion derivatives were subcloned into the pMT vector (Invitrogen) that contains a $\mathrm{CuSO}_{4}$ inducible promoter. In all cases when DIAP1 was expressed in eukaryotic cells, cDNA that encodes C-terminal FLAG-tagged DIAP1 was used (FL DIAP1).

DIAP2 cDNA cloned into pOT2 vector was the generous gift Dr Carl Thummel (Department of Human Genetics and Howard Hughes Medical Institute, University of Utah School of Medicine, Salt Lake City, Utah, USA). For generation of GSTDIAP2, the cDNA was cloned into the pGEX-4T vector (GE Healthcare) and was expressed in BL21 (pLysS) E. coli. Following induction, the protein was affinity purified on glutathione-agarose according to the manufacturer's instructions. C466Y and V453A DIAP2 were generated via site-directed mutagenesis using the GSTDIAP2-coding plasmid. For expression in Drosophila S2 cells, DIAP2 and its mutants were subcloned into the pMT vector (Invitrogen) containing a $\mathrm{CuSO}_{4}$ inducible promoter. cDNA coding for Dronc was cloned in PET14b.

For expression and purification of His-UbcD1, the coding cDNA (cloned in $\mathrm{pET} 14 \mathrm{~b}$ ) was expressed in BL21 (pLysS) E. coli and purified on immobilized $\mathrm{Ni}$ according to the manufacturer's instructions. For purification of GST-Rpr, the corresponding cDNA cloned in pET3a was expressed in BL21 (pLysS) and purified on glutathione-agarose according to the manufacturer's instructions. Plasmids for bacterial expression of various ubiquitin species in which specific lysine residues were substituted with arginine (cloned in pET14b) were obtained from Dr Richard Baer, Colombia University, NY.

Preparation of cell extracts. HeLa cell extract was prepared by hypotonic lysis as described previously. ${ }^{45}$ Reticulocyte-rich blood was induced in rabbits by successive injections of phenylhydrazine, and reticulocyte lysate was prepared as described. ${ }^{46}$

Generation of radiolabeled proteins. $\left.{ }^{35} \mathrm{~S}\right]$ methionine-labeled proteins were synthesized using the in vitro transcription-translation TNT ${ }^{\mathrm{TM}}$ Quick kit.

In vitro processing of DIAP1. In vitro-translated and $\left[{ }^{35}\right.$ S]methionine-labeled DIAP1 was incubated in a reaction mixture containing in a total volume of $12.5 \mu$ : $40 \mathrm{mM}$ Tris- $\mathrm{HCl} \mathrm{pH} 7.6,5 \mathrm{mM} \mathrm{MgCl}, 1 \mathrm{mM} \mathrm{DTT}, 0.05 \mu \mathrm{g}$ hexokinase, $1 \mathrm{mM}$ deoxyglucose and crude cell extract $(50 \mu \mathrm{g})$ as indicated. Staurosporine $(1.5 \mu \mathrm{M})$ was added to the cells (for $4 \mathrm{~h}$ ) prior to their disruption as indicated. Following incubation at $37^{\circ} \mathrm{C}$ for $90 \mathrm{~min}$, reaction mixtures were resolved via sodium dodecyl 
sulfate-polyacrylamide gel electrophoresis (SDS-PAGE; 10\%). Gels were dried, and proteins visualized by Phosphorlmager (Amersham Pharmacia Biosciences)

In vitro degradation of processed DIAP1. Following 90 min incubation for complete caspase-mediated processing of DIAP1 in the absence of ATP (see above), reaction mixtures were further incubated for additional $2 \mathrm{~h}$. The new mixture contained in a total volume of $20 \mu$ : ATP $(2 \mathrm{mM})$, ATP-regenerating system $(20 \mathrm{mM}$ phosphocreatine and $20 \mu \mathrm{g}$ phosphocreatine kinase) and reticulocyte lysate $(2.5 \mu \mathrm{l})$. After boiling, reaction mixtures were resolved via SDS-PAGE, and the gels were dried and exposed to a Phosphorlmager screen.

In vitro ubiquitination. Assays for monitoring in vitro ubiquitination were carried out in reaction mixtures containing in a total volume of $12.5 \mu \mathrm{l}: 40 \mathrm{mM}$ Tris$\mathrm{HCl} \mathrm{pH} \mathrm{7.6,} 5 \mathrm{mM} \mathrm{MgCl}, 1 \mathrm{mM}$ DTT, $5 \mu$ g ubiquitin, $100 \mathrm{ng} \mathrm{UbAl,} 2 \mathrm{mM} \mathrm{ATP} \gamma \mathrm{S}$ and $250 \mathrm{ng} \mathrm{E1} .^{45}$ The reaction mixtures contained also, as indicated, $\left[{ }^{35} \mathrm{~S}\right]$ methioninelabeled WT or mutant DIAP1 ( $40000 \mathrm{cpm}$ ), and $0.5 \mu \mathrm{g} \mathrm{His-UbcD1}$ or $1 \mu \mathrm{g}$ of Ubc13/Uev1a complex. For conjugation of DIAP1 by DIAP2, bacterially purified GST-DIAP2 was added. Reactions were incubated for $50 \mathrm{~min}$ at $37^{\circ} \mathrm{C}$ and resolved via SDS-PAGE $(10 \%)$. Gels were dried and proteins visualized via Phosphorlmaging. When ubiquitin $\mathrm{K}>\mathrm{R}$ mutants were used, and in order to avoid interference of WT ubiquitin present in the translation mixture of the $\left[{ }^{35}\right.$ S]methionine-labeled Myc-tagged DIAP1, the labeled substrate was immunoprecipitated using anti-Myc tag antibody (9E10) immobilized to Protein G. The ubiquitination reaction mixture components were added to the immobilized DIAP1.

Cell transfections and RNA silencing. MCF7 cells (lacking caspase 3) were grown at $37^{\circ} \mathrm{C}$ in Dulbecco's modified Eagle's medium (DMEM) supplemented with $10 \%$ fetal calf serum, insulin $(10 \mu \mathrm{g} / \mathrm{ml})$ and penicillin-streptomycin. Drosophila S2 cells were grown at $24^{\circ} \mathrm{C}$ in Schneider medium (Gibco) supplemented with $10 \%$ fetal calf serum (HyClone) and penicillin-streptomycin. MCF7 cells were transfected using the jetPE| ${ }^{\mathrm{TM}}$ reagent (Polyplus Transfection), and S2 cells were transfected using Cellfectin ${ }^{\mathrm{TM}}$ (Invitrogen) according to the manufacturers' instructions. The RNAi probe ID HFA07444 (GenomeRNAi Drosophila Resources) was used to silence DIAP2 expression. Following PCR, the DNA product was transcribed using the MEGAscript RNAi kit (Ambion). The RNAi $(30 \mu \mathrm{g})$ was added to cells $\left(2 \times 10^{6}\right)$ that were lysed after 48-72 $\mathrm{h}$ in a buffer containing $20 \mathrm{mM}$ HEPES pH 7.4, $1 \mathrm{mM}$ EDTA, $150 \mathrm{mM} \mathrm{NaCl}, 1 \%$ Nonidet P-40, 10\% glycerol, and protease inhibitors. Protein concentration was determined according to the Bradford method using bovine serum albumin as a standard, and equal amounts of protein were resolved via SDS-PAGE (10\%). The gel was blotted onto nitrocellulose membrane, and proteins were detected by ECL after incubation with anti-DIAP1, followed by a secondary antibody.

Processing of DIAP1 in MCF7 cells. MCF7 cells were transfected with $1 \mu \mathrm{g}$ of cDNA coding for FL DIAP1 as described above. Following $30 \mathrm{~h}$, the cells were treated for $4 \mathrm{~h}$ with staurosporine $(1.5 \mu \mathrm{M}), \mathrm{Z}-\operatorname{VAD}(50 \mu \mathrm{M})$ and MG132 $(20 \mu \mathrm{M})$ as indicated, and lysed in a buffer as described above. Equal amounts of protein were resolved via SDS-PAGE $(10 \%)$, and the gel was blotted onto nitrocellulose membrane. Proteins were detected by ECL using anti-FLAG, followed by incubation in the presence of the appropriate secondary antibody.

Stability of DIAP1 in S2 cells. Stability $\left(t_{1 / 2}\right)$ of DIAP1 and its various mutants was monitored in S2 cells following inhibition of protein synthesis by cycloheximide. To express and induce DIAP1, cells were transfected with cDNAs coding for the different species of the enzyme, and after $36 \mathrm{~h}$ were exposed for additional $16 \mathrm{~h}$ to induction medium containing $500 \mu \mathrm{m} \mathrm{CuSO}$. Etoposide $(0.1 \mathrm{mM})$ was added as indicated along with $\mathrm{CuSO}_{4}$. The proteasome inhibitor MG132 $(20 \mu \mathrm{m})$ and the caspase inhibitor Z-VAD $(50 \mu \mathrm{M})$ were added, as indicated, for the last $4 \mathrm{~h}$ of DIAP1 induction. Following induction, cycloheximide $(100 \mu \mathrm{g} / \mathrm{ml})$ was added, and samples were removed and processed at the indicated times. The cells were lysed in a buffer as described above, and equal amounts of protein were resolved via SDS-PAGE (10\%). The gel was blotted onto nitrocellulose membrane, and proteins were detected by ECL using anti-DIAP1 or anti-FLAG antibodies as indicated, followed by incubation in the presence of the appropriate secondary antibody.

DIAP1-DIAP2 binding in vitro. $\left[{ }^{35}\right.$ S $]$ methionine-labeled DIAP2 was incubated with GST-DIAP1 in a $15 \mu \mathrm{l}$ reaction mixture that contained: $40 \mathrm{mM}$
Tris-HCl pH 7.2, $2 \mathrm{mM}$ DTT, $0.2 \mathrm{mg} / \mathrm{ml}$ ovalbumin, $150 \mathrm{mM} \mathrm{NaCl}$ and $0.02 \% \mathrm{NP} 40$. Following incubation at $25^{\circ} \mathrm{C}$ for $30 \mathrm{~min}$, glutathione-agarose beads were added for additional $2 \mathrm{~h}$. The beads were washed four times with the incubation buffer, and the bound proteins were resolved via SDS-PAGE (12\%). Gels were dried and proteins visualized via Phosphorlmaging.

Acknowledgements. We thank Dr Kazuhiro Iwai (City of Osaka University School of Medicine, Osaka, Japan) for his help in subcloning some of the cDNAs. Research in the laboratory of $A C$ is supported by grants from the Prostate Cancer Foundation Israel - Centers of Excellence Program, the Israel Science Foundation (ISF), the European Union (EU) Networks of Excellence (NeOEs) on Neurodegeneration (NeuroNE) and Ubiquitin and Ubiquitin-Like Modifiers (RUbicon), the Foundation for Promotion of Research in the Technion, and a Professorship funded by the Israel Cancer Research Fund (ICRF) USA. Infrastructural equipment has been purchased with the support of the Wolfson Charitable Fund Center of Excellence for studies on Turnover of Cellular Proteins and its Implications to Human Diseases.

1. Hay BA, Wassarman DA, Rubin GM. Drosophila homologs of Baculovirus inhibitor of apoptosis proteins function to block cell death. Cell 1995; 83: 1253-1262.

2. Vaux DL, Silke J. IAPs, RINGs and ubiquitylation. Nat Rev Mol Cell Biol 2005; 6: 287-297.

3. Goyal L, McCall K, Agapite J, Hartwieg E, Steller H. Induction of apoptosis by Drosophila reaper, hid and grim through inhibition of IAP function. EMBO J 2000; 19: 589-597.

4. Wang SL, Hawkins CJ, Yoo SJ, Muller HA, Hay BA. The Drosophila caspase inhibitor DIAP1 is essential for cell survival and is negatively regulated by HID. Cell 1999; 98: 453-463.

5. Lisi S, Mazzon I, White K. Diverse domains of THREAD/DIAP1 are required to inhibit apoptosis induced by REAPER and HID in Drosophila. Genetics 2000; 154: 669-678.

6. Wu JW, Cocina AE, Chai J, Hay BA, Shi Y. Structural analysis of a functional DIAP1 fragment bound to grim and hid peptides. Mol Cell 2001; 8: 95-104.

7. Fang S, Weissman AM. A field guide to ubiquitylation. Cell Mol Life Sci 2004; 61: 1546-1561.

8. Glickman $\mathrm{MH}$, Ciechanover A. The ubiquitin-proteasome proteolytic pathway: destruction for the sake of construction. Physiol Rev 2002; 82: 373-428.

9. Ryoo HD, Bergmann A, Gonen H, Ciechanover A, Steller H. Regulation of Drosophila IAP1 degradation and apoptosis by reaper and UbcD1. Nat Cell Biol 2002; 4: 432-438.

10. Martin SJ. Destabilizing influences in apoptosis: sowing the seeds of IAP destruction. Cell 2002; 109: 793-796.

11. Yoo SJ, Huh JR, Muro I, Yu H, Wang L, Wang SL et al. Hid, Rpr and Grim negatively regulate DIAP1 levels through distinct mechanisms. Nat Cell Biol 2002; 4: 416-424.

12. Chai J, Yan N, Huh JR, Wu JW, Li W, Hay BA et al. Molecular mechanism of Reaper-GrimHid-mediated suppression of DIAP1-dependent Dronc ubiquitination. Nat Struct Biol 2003 10: 892-898.

13. Kuranaga E, Kanuka H, Igaki T, Sawamoto K, Ichijo H, Okano H et al. Reaper-mediated inhibition of DIAP1-induced DTRAF1 degradation results in activation of JNK in Drosophila. Nat Cell Biol 2002; 4: 705-710.

14. Wilson R, Goyal L, Ditzel M, Zachariou A, Baker DA, Agapite J et al. The DIAP1 RING finger mediates ubiquitination of Dronc and is indispensable for regulating apoptosis. Nat Cell Biol 2002; 4: 445-450.

15. Olson MR, Holley CL, Yoo SJ, Huh JR, Hay BA, Kornbluth S. Reaper is regulated by IAPmediated ubiquitination. J Biol Chem 2003; 278: 4028-4034.

16. Wing J, Zhou L, Schwartz L, Nambu J. Distinct cell killing properties of the Drosophila reaper, head involution defective, and grim genes. Cell Death Differ 1999; 6: 212-213.

17. Kaiser WJ, Vucic D, Miller LK. The Drosophila inhibitor of apoptosis D-IAP1 suppresses cell death induced by the caspase DrICE. FEBS Lett 1998; 440: 243-248.

18. Doumanis J, Quinn L, Richardson H, Kumar S. STRICA, a novel Drosophila melanogaster caspase with an unusual serine/threonine-rich prodomain, interacts with DIAP1 and DIAP2. Cell Death Differ 2001; 8: 387-394.

19. Ditzel M, Wilson R, Tenev T, Zachariou A, Paul A, Deas E et al. Degradation of DIAP1 by the $\mathrm{N}$-end rule pathway is essential for regulating apoptosis. Nat Cell Biol 2003; 5: 467-473.

20. Bachmair A, Finley D, Varshavsky A. In vivo half-life of a protein is a function of its aminoterminal residue. Science 1986; 234: 179-186.

21. Stewart $A E$, Arfin $\mathrm{SM}$, Bradshaw RA. Protein $\mathrm{NH}_{2}$-terminal asparagine deamidase. Isolation and characterization of a new enzyme. J Biol Chem 1994; 269: 23509-23517.

22. Varshavsky A. The N-end rule: functions, mysteries, uses. Proc Natl Acad Sci USA 1996; 93: 12142-12149.

23. Baker RT, Varshavsky A. Yeast $\mathrm{N}$-terminal amidase. A new enzyme and component of the $\mathrm{N}$-end rule pathway. J Biol Chem 1995; 270: 12065-12074.

24. Ciechanover A, Ferber S, Ganoth D, Elias S, Hershko A, Arfin S. Purification and characterization of arginyl-tRNA-protein transferase from rabbit reticulocytes. Its involvement in post-translational modification and degradation of acidic $\mathrm{NH}_{2}$ termin substrates of the ubiquitin pathway. J Biol Chem 1988; 263: 11155-11167.

25. Ferber $\mathrm{S}$, Ciechanover $\mathrm{A}$. Role of arginine-tRNA in protein degradation by the ubiquitin pathway. Nature 1987; 326: 808-811. 
26. Yokokura T, Dresnek D, Huseinovic N, Lisi S, Abdelwahid E, Bangs $\mathrm{P}$ et al. Dissection of DIAP1 functional domains via a mutant replacement strategy. J Biol Chem 2004; 279 52603-52612.

27. Yan N, Wu JW, Chai J, Li W, Shi Y. Molecular mechanisms of DrICE inhibition by DIAP1 and removal of inhibition by Reaper, Hid and Grim. Nat Struct Mol Biol 2004; 11: 420-428.

28. Deveraux QL, Leo E, Stennicke HR, Welsh K, Salvesen GS, Reed JC. Cleavage of human inhibitor of apoptosis protein XIAP results in fragments with distinct specificities for caspases. EMBO J 1999; 18: 5242-5251.

29. Clem RJ, Sheu TT, Richter BW, He WW, Thomberry NA, Duckett CS et al. cIAP1 is cleaved by caspases to produce a proapoptotic C-terminal fragment. J Biol Chem 2001; 276: 7602-7608.

30. Hershko A, Heller H. Occurrence of a polyubiquitin structure in ubiquitin-protein conjugates Biochem Biophys Res Commun 1985; 128: 1079-1086.

31. Reiss $\mathrm{Y}, \mathrm{Kaim} \mathrm{D}$, Hershko A. Specificity of binding of $\mathrm{NH}_{2}$-terminal residue of proteins to ubiquitin-protein ligase. Use of amino acid derivatives to characterize specific binding sites. J Biol Chem 1988; 263: 2693-2698.

32. Bachmair A, Varshavsky A. The degradation signal in a short-lived protein. Cell 1989; 56 1019-1032.

33. Brzovic PS, Keeffe JR, Nishikawa H, Miyamoto K, Fox III D, Fukuda M et al. Binding and recognition in the assembly of an active BRCA1/BARD1 ubiquitin-ligase complex. Proc Natl Acad Sci USA 2003; 100: 5646-5651.

34. Muro I, Means JC, Clem RJ. Cleavage of the apoptosis inhibitor DIAP1 by the apical caspase DRONC in both normal and apoptotic Drosophila cells. J Biol Chem 2005; 280: 18683-18688.

35. Hofmann RM, Pickart CM. Noncanonical MMS2-encoded ubiquitin-conjugating enzyme functions in assembly of novel polyubiquitin chains for DNA repair. Cell 1999; 96: 645-653.

36. Andersen PL, Zhou H, Pastushok L, Moraes T, McKenna S, Ziola B et al. (2005) Distinct regulation of $\mathrm{Ubc13}$ functions by the two ubiquitin-conjugating enzyme variants Mms2 and Uev1A. J Cell Biol 2005; 170: 745-755.
37. Geetha T, Jiang J, Wooten MW. Lysine 63 polyubiquitination of the nerve growth factor receptor TrkA directs internalization and signaling. Mol Cell 2005; 20: 301-312.

38. Adhikary S, Marinoni F, Hock A, Hulleman E, Popov N, Beier R et al. The ubiquitin ligase HectH9 regulates transcriptional activation by Myc and is essential for tumor cell proliferation. Cell 2005; 123: 409-421.

39. Zhou R, Silverman N, Hong M, Liao DS, Chung Y, Chen ZJ et al. The role of ubiquitination in Drosophila innate immunity. J Biol Chem 2005; 280: 34048-34055.

40. Silke J, Kratina T, Chu D, Ekert PG, Day CL, Pakusch M et al. Determination of cell survival by RING-mediated regulation of inhibitor of apoptosis (IAP) protein abundance. Proc Natl Acad Sci USA 2005; 102: 16182-16187.

41. Deng L, Wang C, Spencer E, Yang L, Braun A, You J et al. Activation of the $\mathrm{I}_{\kappa} \mathrm{B}$ kinase complex by TRAF6 requires a dimeric ubiquitin-conjugating enzyme complex and a unique polyubiquitin chain. Cell 2000; 103: 351-361.

42. Wing JP, Schreader BA, Yokokura T, Wang Y, Andrews PS, Huseinovic N et al. Drosophila Morgue is an $\mathrm{F}$ box/ubiquitin conjugase domain protein important for grim-reaper mediated apoptosis. Nat Cell Biol 2002; 4: 451-456.

43. Hays R, Wickline L, Cagan R. Morgue mediates apoptosis in the Drosophila melanogaster retina by promoting degradation of DIAP1. Nat Cell Biol 2002; 4: 425-431.

44. Kuranaga EKH, Tonoki A, Takemoto K, Tomioka T, Kobayashi M, Hayashi S et al. Drosophila IKK-related kinase regulates nonapoptotic function of caspases via degradation of IAPs. Cell 2006; 126: 583-596.

45. Gonen H, Bercovich B, Orian A, Carrano A, Takizawa C, Yamanaka K et al. Identification of the ubiquitin carrier proteins, E2s, involved in signal-induced conjugation and subsequent degradation of $\mathrm{I}_{\kappa} \mathrm{B} \alpha$. J Biol Chem 1999; 274: 14823-14830.

46. Hershko A, Heller $\mathrm{H}$, Elias S, Ciechanover A. Components of ubiquitin-protein ligase system. Resolution, affinity purification, and role in protein breakdown. J Biol Chem 1983; 258: $8206-8214$ 